\title{
Du tutoiement et du vouvoiement
}

\section{Eberhard Wolff}

Prof. Dr rer. soc., rédacteur culture, histoire, société

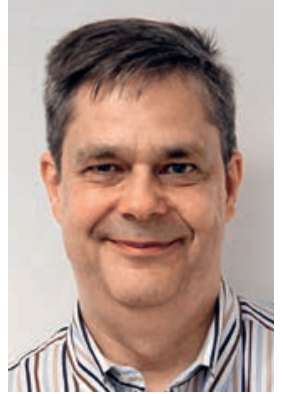

Lorsque je suis venu d'Allemagne à Zurich sur une très mince plaque de glace professionnelle il y a vingt ans, mes collègues, y compris ceux qui occupaient des postes plus élevés, m'ont rapidement proposé le tutoiement. Cela a facilité mon arrivée. Ils ne l'ont toutefois pas fait sans souligner avec fierté au passage que les hiérarchies étaient plus plates en Suisse qu'en Allemagne. Surtout dans les hôpitaux, qui accueillaient à l'époque de plus en plus d'Allemands. Mon intégration par le tutoiement était aussi une manière de se démarquer «des autres», de ceux du Nord.

De fait, j'ai en mémoire une situation curieuse avec un nouveau collègue qui m'était sympathique, alors que j'étais jeune boursier dans un institut de recherche allemand. Aucun de nous n'a proposé le tutoiement à l'autre. Moi, parce qu'il avait déjà son doctorat et un "vrai» poste et lui, parce que j'étais plus âgé. Nous nous sommes vouvoyés durant des semaines, bien qu'aucun de nous deux ne le veuille réellement. Les règles de bienséance étaient compliquées et nous n'étions ni l'un ni l'autre très sûrs de nous. A la suite d'une promenade, nous nous sommes appelés par nos prénoms.

Le tutoiement général entre professionnels, des médecins aux assistantes médicales en passant par les apprentis, s'est probablement imposé dans de nombreux cabinets médicaux suisses. Cela crée sans aucun doute une certaine cohésion, mais les hiérarchies et les différences n'en sont pas simplement éliminées pour autant. Une grande entreprise suisse active à l'international a récemment introduit le tutoiement général entre collègues. La méthode IKEA, pour ainsi dire. Mais ce qui se voulait un moyen de créer une ambiance de travail ouverte ne convenait pas à tout le monde. Dire simplement «tu» au CEO auréolé de toute sa puissance? Cela ne cadre pas vraiment avec la culture suisse. (Oups, me dis-je, n'est-ce pas contraire à ce que l'on vient d'affirmer sur la manière dont les Suisses s'abordent entre eux?)

En tout cas, le tutoiement imposé d'en haut peut aussi être source de tensions. Moi-même, je préfère vouvoyer certaines personnes, avec tout le respect qui leur est dû, que les tutoyer.

Contrairement à mes collègues plus jeunes, je vouvoie aussi les étudiants de mes séminaires universitaires. La distance ainsi instaurée est volontaire. Même s'il semble souvent guindé, le vouvoiement reflète à mon sens mieux les différences très réelles que le «tu» géné- ralisé. A mes yeux, c'est aussi une marque de respect vis-à-vis des jeunes étudiants.

Wolf Biermann, le parolier, chanteur et critique du système de l'Allemagne de l'Est socialiste, a indiqué dans les années 70 qu'il savait à l'époque très vite si les fans qui sonnaient à sa porte venaient de la RDA ou de la RFA. Ceux de l'Ouest lui disaient "tu», ceux de l'Est «vous». A l'Est, les camarades se tutoyaient. A l'Ouest, on commençait par se dire "vous». Dans les deux cas, les fans utilisaient la formule de salutation comme un petit signe de rébellion solidaire face à la pratique imposée dans leur Etat.

Le tutoiement général va de soi dans de nombreux pays. En anglais, il n'existe qu'une manière de s'adresser aux autres: "you». Ce n'est pas pour autant synonyme d'une absence de hiérarchie. Dans la célèbre série télévisée Breaking Bad, dans laquelle le professeur de chimie Walter White devient fabricant puis caïd de la drogue, son jeune compère Jesse Pinkman s'adresse à lui jusqu'au bout en l'appelant «Mister White»: il a été son élève en cours de chimie. Et Mr. White appelle Jesse par son prénom.

Dans les hôpitaux suisses, la manière dont les collaborateurs s'adressent les uns aux autres est sans doute nettement plus complexe que dans les cabinets médicaux à taille humaine. Si vous y travaillez, essayez de déterminer où se situe la limite du vouvoiement: "dès» le niveau des chefs de clinique ou "seulement» à l'échelon des médecins chefs? Qu'en est-il vis-à-vis des collègues non-médecins? Tutoyez-vous les membres de l'équipe de nettoyage et êtes-vous tutoyés par eux? Les femmes se tutoient-elles plus vite entre elles que les hommes? Comment cela se passe-t-il entre les sexes? Une TRM m'a parlé d'un collègue médecin proche qu'elle tutoyait. Lorsqu'il est revenu dans son ancien hôpital en tant que médecin chef après avoir exercé ailleurs, le vouvoiement est devenu la règle et le «tu» d'autrefois a posé un véritable problème.

Même si nous nous tutoyons de plus en plus, la manière de s'adresser aux autres reste toujours compliquée, pour la bonne et simple raison que nos relations le sont aussi. Et je n'ai même pas évoqué les formules des patients (qui vont de "Docteur Hefti» en parlant au médecin à l'inévitable «Regula» en s'adressant à l'infirmière).

Plus j'y songe, plus j'apprécie le classique vouvoiement. N'es-tu pas de mon avis, chère lectrice, cher lecteur? 